\title{
Carcinoma mamario en un canino macho: caracterización clínica e inmunohistoquímica
}

\author{
Mammary carcinoma in a male dog: \\ clinical and immunohistochemical characterisation \\ JI Arias ${ }^{\mathrm{a}}$, E Paredes $^{\mathrm{b}}$, CG Torres ${ }^{\mathrm{a}}$ \\ aDepartamento de Ciencias Clínicas, Facultad de Ciencias Veterinarias y Pecuarias, \\ Universidad de Chile, Santiago, Chile. \\ bInstituto de Patología Animal, Facultad de Ciencias Veterinarias, \\ Universidad Austral de Chile, Valdivia, Chile.
}

\begin{abstract}
SUMMARY
Mammary tumors in male dogs are rare, not exceeding $2 \%$ of all cases of mammary tumors in males and females. These have proved to be mostly of low-grade malignancy and positive for the presence of estradiol receptor $\alpha$. In this case report, a male mammary cancer in a mixed breed dog is shown. This case report presents a case or mammary tumor which was clinically evaluated and surgically resolved, it was also under cytological, histological and immunohistochemical assessment, by studying proteins such as estradiol receptor $\alpha$ and $\beta$ (ER $\alpha$, RE $\beta$ ) epidermal growth factor receptor 2 (EGFR2) vascular endothelial growth factor receptor 2 (VEGFR2), cyclooxygenase-2 (COX2) and proliferating cell nuclear antigen (PCNA). Clinical examination showed that the patient had a well-defined mass, filled with liquid, adjacent to the prepuce and forming part of the right inguinal mammary gland. Cytology and histology showed neoplastic epithelial cells, consistent with a tubular carcinoma simple type, histological grade I. When performing immunedetection, high expression of RE $\alpha, R E \beta$ and EGFR2 and absence of expression of COX2 and VEGFR2 were detected. These immunological results are consistent with the clinical and histological finds of the studied tumor.
\end{abstract}

Key words: mammary carcinoma, male dog, estradiol receptor.

RESUMEN

\begin{abstract}
Las neoplasias mamarias en perros machos son poco comunes, no superando el $2 \%$ de la totalidad de casos de tumores mamarios en machos y hembras. Estos han mostrado ser en su mayoría de baja malignidad y positivos a la presencia del receptor de estradiol $\alpha$. En este reporte se presenta un caso de tumor mamario en un perro mestizo macho, que fue evaluado clínicamente, resuelto quirúrgicamente y estudiado citológica, histológica e inmunohistoquimicamente mediante el estudio de proteínas como receptor de estradiol $\alpha$ y $\beta$ (RE $\alpha$, RE $\beta$ ), receptor 2 del factor de crecimiento epidérmico (EGFR2), receptor 2 del factor de crecimiento vascular endotelial (VEGFR2), ciclooxigenasa 2 (COX2) y antígeno nuclear de proliferación celular (PCNA). Al examen clínico, el paciente presentó una masa bien definida, llena de líquido, adyacente al prepucio y formando parte de la glándula mamaria inguinal derecha. La citología y el estudio histológico mostraron células epiteliales neoplásicas concordantes con un carcinoma tubular simple de grado histológico I. Al realizar inmunodetección, se observó una alta expresión de RE $\alpha$, RE $\beta$ y EGFR2 y ausencia de expresión de COX2 y VEGFR2, resultados que son concordantes con el patrón clínico-histológico de la neoplasia estudiada.
\end{abstract}

Palabras clave: carcinoma mamario, perro macho, receptor de estradiol.

\section{INTRODUCCIÓN}

Los tumores de glándula mamaria en caninos son comúnmente diagnosticados en la clínica diaria (Moe 2001, Richards y col 2001). Estos representan alrededor del 50\% de los tumores que se producen en las perras (Zatloukal y col 2005), siendo esta incidencia tres veces superior a la reportada en mujeres (Caicedo y col 2012). La mayoría de los tumores de la glándula mamaria en perras son de origen epitelial, y aproximadamente el

Aceptado: 10.07.2014.

* crtorres@uchile.cl
50\% son malignos (Sleeckx y col 2011). El cáncer de mama humano es poco frecuente de ser encontrado en el hombre, representando menos del $1 \%$ del total de casos diagnosticados (Crichlow y Galt 1990). Por lo general se detectan en hombres mayores y los factores de riesgo más probables para esta enfermedad incluyen aumento de la edad, lesiones testiculares, obesidad, ginecomastia, síndrome de Klinefelter y una historia familiar de cáncer de mamas (Bhagat y Kline 1990, Crichlow y Galt 1990, Demeter 1990). La mayoría de estos tumores son malignos, y aproximadamente el 65-80\% expresan el receptor de estradiol tipo $\alpha$ (Bezwoda y col 1987). Lo anterior hace que estos tumores en el hombre sean asociados a alteraciones en el equilibrio de estrógenos y andrógenos, con un mayor riesgo en pacientes con testículos no descendidos, 
orquitis, daño testicular o en alteraciones que conllevan un estado de hiperestrogenismo (Giordano y col 2002).

En el caso de los perros machos se repite esta estadística, presentándose en forma poco frecuente. Es así como Mulligan (1975), al hacer una revisión de 120 casos de tumores mamarios en caninos, solo encontró dos casos en machos (1,66\%). Unos años después Priester y McKay (1980) estudiaron 1.195 casos de neoplasias mamarias malignas confirmadas histológicamente, y solo encontraron 13 machos afectados $(1,08 \%)$, mientras que de 562 neoplasias mamarias benignas, solo 10 ocurrieron en machos (1,7\%) (Priester y McKay 1980). Saba y col (2007) analizaron tumores mamarios provenientes de ocho perros machos, los que mostraron una alta expresión del receptor de estrógeno $\alpha$. Por otra parte, la expresión del receptor de progesterona, aunque estaba presente en todos los tumores, fue de menor intensidad.

El objetivo de esta comunicación es caracterizar clínica e inmunohistoquimicamente un caso de neoplasia mamaria en un perro macho.

\section{MATERIAL Y MÉTODOS}

\section{CASO CLÍNICO}

En el Hospital Clínico Veterinario de la Facultad de Ciencias Veterinarias de la Universidad Austral de Chile se presentó un perro macho entero, mestizo, de 10 años de edad, que presentaba una masa de $10 \mathrm{~cm}$ de diámetro llena de líquido, adyacente al prepucio y formando parte de la glándula mamaria inguinal derecha. Se realizó examen clínico general, hematología, bioquímica sanguínea y radiografías de tórax como métodos diagnósticos complementarios. El paciente fue estadificado clínico-patológicamente según lo descrito por Cassali y col (2011). Posteriormente, el paciente fue sometido a cirugía (mastectomía inguinal derecha) bajo anestesia general inhalatoria y técnica quirúrgica aséptica.

\section{CITOLOGÍA, HISTOPATOLOGÍA E INMUNOHISTOQUÍMICA}

Se obtuvieron muestras mediante punción con aguja fina y drenaje del tumor ya extraído quirúrgicamente para realizar un examen citológico del mismo. Esta muestra fue directamente extendida sobre portaobjetos y secada al aire para luego ser teñida con tinción Diff- Quick®.
Por otra parte, se obtuvieron muestras del tejido mamario extirpado, siendo fijadas en formalina tamponada al 10\%, posteriormente fueron deshidratadas e incluidas en parafina mediante técnicas rutinarias. Los cortes de $4 \mu \mathrm{m}$ así obtenidos, fueron teñidos con tinción H\&E para su caracterización histológica. El tipo y graduación histológica se realizó de acuerdo con lo descrito por Goldschmidt y col (2011). Para caracterizar molecularmente la neoplasia en estudio se realizó análisis inmunohistoquímico del receptor de estradiol $\alpha$ y $\beta$ (RE $\alpha, \operatorname{RE} \beta)$, receptor 2 del factor de crecimiento epidérmico (EGFR2), receptor 2 del factor de crecimiento vascular endotelial (VEGFR2), ciclooxigenasa 2 (COX2) y antígeno nuclear de proliferación celular (PCNA) (Torres y col 2009). Brevemente, después que los cortes histológicos fueron desparafinados en Neoclear (Merck) y rehidratados en etanol, se realizó recuperación antigénica sumergiendo los cortes en buffer citrato $0,01 \mathrm{M}$, $\mathrm{pH} 6.0$ y fueron hervidos durante 40 minutos en vaporera. Las muestras fueron sometidas a bloqueo de peroxidasa endógena en $\mathrm{H}_{2} \mathrm{O}_{2}$ al $10 \%$ durante 30 minutos para posteriormente mantenerse durante 15 minutos en una solución de albúmina sérica bovina (BSA) $2 \%$ en TBS. Se realizó incubación con anticuerpos primarios (cuadro 1) durante toda la noche $\mathrm{a} 4^{\circ} \mathrm{C}$. Para la reacción inmunohistoquímica se utilizó el kit ImmPress ${ }^{\mathrm{TM}}$ Universal Antibody (Vector Laboratories) (anticuerpos secundarios anti-IgG de ratón / anti-IgG de conejo conjugados con un complejo polímero/peroxidasa). Para evidenciar la reacción se utilizó el sustrato 3,3'-Diaminibenzidina (DAB) y contratinción con Hematoxilina de Mayer. Se consideraron controles negativos donde se realizó incubación con solución de BSA $2 \%$ en vez del anticuerpo primario.

\section{RESULTADOS Y DISCUSIÓN}

Clínicamente, el tumor mamario se mostró bien delimitado, de base pedunculada, de aspecto fluctuante, indoloro y de crecimiento no invasivo (figura 1a), características que son propias de una neoplasia de baja malignidad (Sorenmo 2003). El examen clínico general y las pruebas complementarias no revelaron alteraciones que sugieran cambios paraneoplásicos (datos no mostrados), estadificándose la neoplasia en estadio clínico III. Luego de la mastectomía regional, el tumor fue diseccionado constatando la presencia de un líquido sero-sanguinolento con un volumen aproximado de $200 \mathrm{ml}$; esto demostró la naturaleza quística

Cuadro 1. Anticuerpos primarios utilizados en el estudio inmunohistoquímico.

Primary antibodies used in the immunohistochemical study.

\begin{tabular}{lcccccc}
\hline Anticuerpo $1^{\circ}$ & RE $\alpha$ & RE $\beta$ & EGFR2 & COX2 & VEGFR2 & PCNA \\
\hline Especie & conejo & conejo & conejo & conejo & ratón & ratón \\
Dilución & $1: 100$ & $1: 100$ & $1: 200$ & $1: 50$ & $1: 100$ & $1: 200$ \\
Fuente & SCBT & SCBT & SCBT & Thermo Scientific & SCBT & Thermo Scientific \\
\hline
\end{tabular}




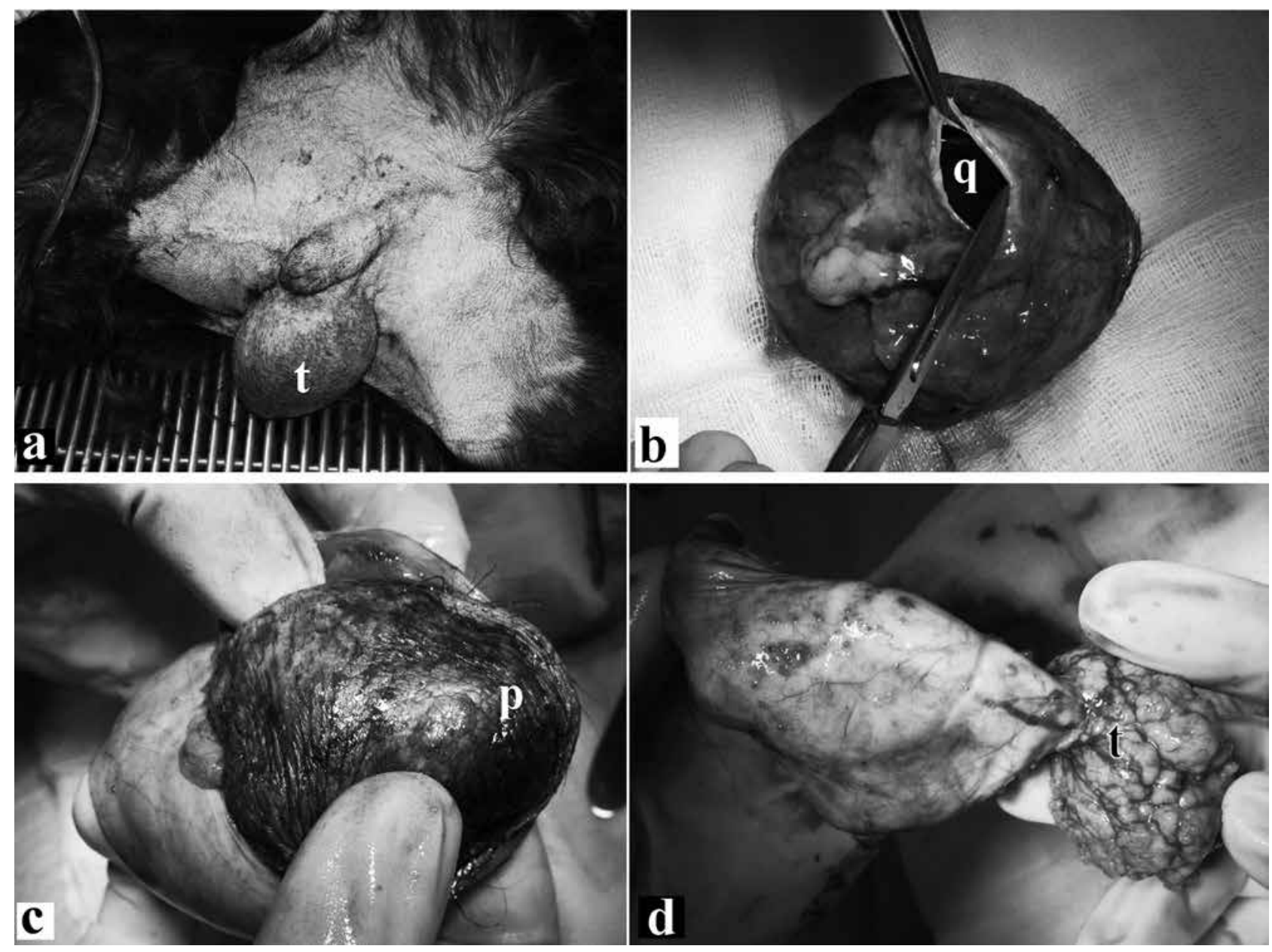

Figura 1. Fotografías prequirúrgicas e intraoperatorias del tumor mamario. (a) se observa el tamaño y ubicación de la masa tumoral (t); (b) se muestra el tumor quístico (q) ya extraído con su contenido sero-sanguinolento; (c) muestra la superficie de la piel extirpada y la ubicación del pezón de la mama inguinal (p); (d) muestra la morfología del tumor (t) por debajo del pezón afectado.

Prequirurgical and intraoperatory mammary tumor photographs. (a) the size and location of the tumoral mass (t) can be observed; (b) cystic tumor (q) already excised with its serosanguinolent content; (c) the surface of the excised skin and the location of the inguinal mammary nipple (p) is shown; (d) the morphology of the tumor (t) under de affected nipple is shown.

de la neoplasia (figura 1b). El tumor involucraba al pezón de la mama inguinal derecha (figura 1c), desarrollándose probablemente desde el ducto mamario hacia el parénquima glandular. Al drenar completamente la masa quística original se observó que el tamaño real del tumor era de $4 \mathrm{~cm}$ en su eje principal (figura 1d). Considerando lo anterior, la neoplasia se reestadificó en estadio clínico II (Cassali y col 2011).

En el diagnóstico de la neoplasia mamaria no se recomienda el aspirado con aguja fina para citología debido a que es un método de baja sensibilidad (Sorenmo 2003). No obstante ya que la masa original era quística, se consideró relevante hacer un análisis morfológico del sedimento celular del líquido intratumoral. La citología mostró presencia de abundantes células epiteliales de aspecto neoplásico dispuestas en grupos cohesivos. Estas células tenían alta relación núcleo-citoplasma, pleomorfismo y anisocitosis leve a moderada, presencia de nucléolos prominentes y citoplasma basofílico, todas características de malignidad (figura 2a). Debido a lo anterior y pese a lo descrito por la literatura pertinente, se sugiere que hacer citología de masas tumorales de aspecto quístico podría ayudar a orientar el diagnóstico definitivo.

Histológicamente, las secciones de la neoplasia mamaria mostraron un tejido de neoformación bien delimitado, no encapsulado, compuesto por células epiteliales neoplásicas dispuestas en nidos y cordones con formación de abundantes túbulos, algunos de ellos con presencia de material eosinofílico en su lumen, rodeados por un estroma fibrovascular en moderada cantidad. Las células tumorales mostraron atipia nuclear leve a moderada y escasa actividad mitótica. El estudio histopatológico fue concordante con un carcinoma tubular simple de grado histológico I (figura 2b) según lo descrito por Goldschmidt y col (2011). Al respecto, Sorenmo (2003) y Cassali y col (2011) describen que los carcinomas tubulares de bajo grado histológico, pese a ser 

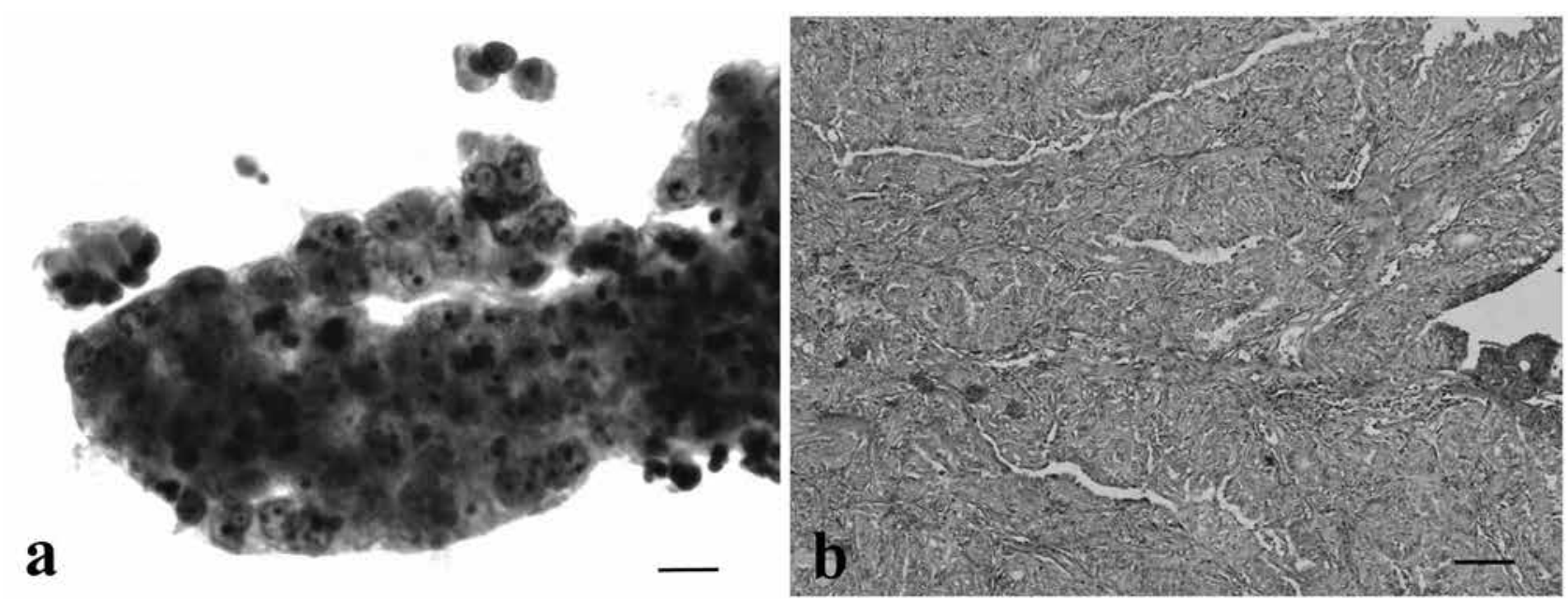

Figura 2. Citología y sección histológica del tumor mamario. (a) Citología del líquido intratumoral muestra abundantes acúmulos de células epiteliales de aspecto neoplásico. Diff- Quick®, barra corresponde a $20 \mu \mathrm{m}$; (b) Corte histológico muestra la presencia de tejido neoproliferativo de base epitelial con formación de abundantes túbulos, compatibles con carcinoma tubular simple de grado histológico I. Hematoxilina \& Eosina, barra corresponde a $100 \mu \mathrm{m}$.

Mammary tumor cytology and histological section. (a) Intratumoral liquid cytology showing abundant epithelial cells clusters of neoplastic aspect. Diff- Quick®, bar corresponds to $20 \mu \mathrm{m}$; (b) Histological section shows the presence of epithelial based neoproliferative tissue with abundant tubules, compatible with histological grade I carcinoma simple tubular Type. Hematoxilina \& Eosina, bar corresponds to $100 \mu \mathrm{m}$.

de naturaleza histológica maligna, tienen bajo potencial de reaparición local y metástasis, lo que se traduce en una alta probabilidad de tiempos de sobrevida prolongados posterior al manejo quirúrgico.

Para determinar el pronóstico se hizo un análisis inmunohistoquímico estudiando proteínas cuya presencia se asocian a buen (RE $\alpha, \mathrm{RE} \beta)$ (Millanta y col 2005) y mal pronóstico (COX2 y VEGFR2) (Doré 2011, Camacho y col 2014). Se observó una alta presencia del RE $\alpha$ (figura 3b) y RE $\beta$, expresión que se visualizó tanto a nivel nuclear como citoplasmático de las células neoplásicas. EGFR2 se inmunodetectó en el citoplasma de la mayoría de las células neoplásicas que formaban parte del tumor estudiado (figura 3c). No se observó expresión de COX2 (figura 3d) ni de VEGFR2 (no mostrado). Con el fin de establecer más específicamente la tasa proliferativa de esta neoplasia, se estudió proliferación celular mediante la inmunodetección de PCNA, biomarcador que se expresa en todas las fases del ciclo celular a excepción de G0 donde se expresa escasamente (Vinothini y col 2009). Así, la expresión de PCNA fue baja a moderada $(17,5 \pm 4,1 \%)$. Estos resultados inmunohistoquímicos son concordantes con una neoplasia de baja malignidad y corroboran las observaciones clínicas e histológicas ya descritas. La baja malignidad observada en este caso es congruente con lo descrito por Bearss y col (2012), quienes caracterizaron 27 casos de neoplasia mamaria en perros machos, cuyos patrones histológicos fueron benignos. Recientemente, Im y col (2014) describieron una nueva clasificación molecular para tumores mamarios en perros basado en una clasificación utilizada en las neoplasias mamarias humanas, la cual tiene valor pronóstico y orientaría de forma más específica el tratamiento. El tumor mamario aquí descrito se clasificaría molecularmente como Luminal B debido a su patrón de expresión positivo para RE $\alpha$ y EGFR2, patrón que se presenta fundamentalmente en carcinomas de grado histológico bajo y moderado (Im y col 2014).

\section{REFERENCIAS}

Bearss JJ, FY Schulman, D Carter. 2012. Histologic, immunohistochemical, and clinical features of 27 mammary tumors in 18 male dogs. Vet Pathol 49, 602-607.

Bezwoda WR, C Hesdorffer, R Dansey, N De Moor, DP Derman, S Browde, M Lange. 1987. Breast cancer in men: Clinical features, hormone receptor status, and response to therapy. Cancer 60, 1337-1340.

Bhagat P, TS Kline. 1990. The male breast and malignant neoplasms: Diagnosis by aspiration biopsy cytology. Cancer 65, 2338-2341.

Caicedo JA, CA Iregui, ME Cabarcas, BJ Acosta. 2012. Estudio comparativo de la frecuencia de tumores mamarios según sexo, edad y tipo histológico en caninos y humanos en los laboratorios de patología anatómica de la Universidad Nacional de Colombia sede Bogotá. Revista Colombiana de Ciencia Animal 5, 37-46.

Camacho L, L Peña, AG Gil, A Martín-Ruiz, S Dunner, JC Illera. 2014. Immunohistochemical vascular factor expression in canine inflammatory mammary carcinoma. Vet Pathol 51, 737-748.

Cassali GD, GE Lavalle, AB De Nardi, E Ferreira, AC Bertagnolli, A Estrela-Lima, AC Alessi, CR Daleck, B Salgado, CG Fernandes, RA Sobral, RL Amorim, CO Gamba, KA Damasceno, PA Auler, GM Magalhães, J Silva, JB Raposo, AMR Ferreira, LO Oliveira, C Malm, DAPC Zuccari, NM Tanaka, LR Ribeiro, LC Campos, CM Souza, J Leite, LMC Soares, MF Cavalcanti, ZGC Fonteles, ID Schuch, J Paniago, TS Oliveira, EM Terra, TLL Castanheira, AOC Felix, GD Carvalho, TN Guim, TN Guim, E Garrido, SC Fernandes, FCL Maia, MLZ Dagli, NS Rocha, H Fukumasu, F Grandi, JP Machado, SMMS Silva, JE Bezerri, MS Frehse, EC Paes de Almeida, CB Campos. 2011. Consensus for the diagnosis, prognosis and treatment of canine mammary tumors. Braz J Vet Pathol 4, 153-180. 

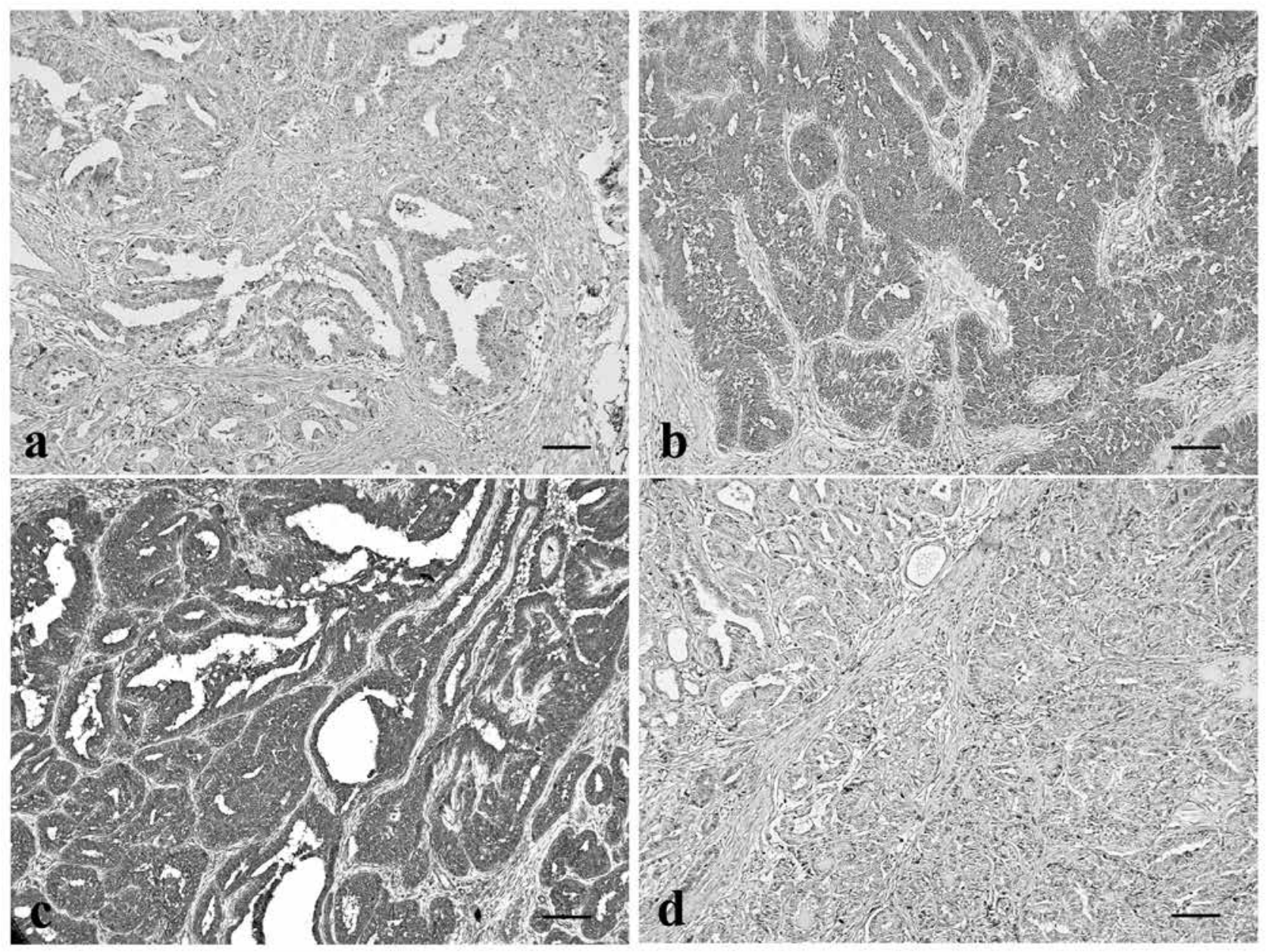

Figura 3. Imágenes inmunohistoquímicas representativas de la neoplasia mamaria. La inmunotinción positiva (nuclear o citoplasmática)

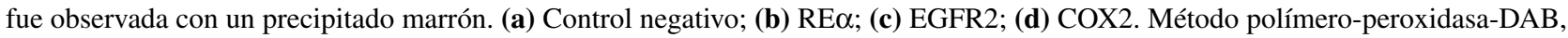
barra corresponde a $100 \mu \mathrm{m}$.

Immunohistochemical representative images of mammary tumor. The positive immunostaining (nuclear or cytoplasmatic) was observed as brown precipitate. (a) Negative control, (b) ER $\alpha$, (c) EGFR2, (d) COX2. Method polymer-peroxidase-DAB, bar corresponds to $100 \mu \mathrm{m}$.

Crichlow RW, SW Galt. 1990. Male breast cancer. Surg Clin North Am 70, 1165-1177.

Demeter JG, NG Watermen, GD Verdi. 1990. Familial male breast carcinoma. Cancer 65, 2542-2543.

Doré M. 2011. Cyclooxygenase-2 expression in animal cancers. Vet Pathol 48, 254-265.

Giordano SH, AU Buzdar, GN Hortobagyi. 2002. Breast cancer in men. Ann Intern Med 137, 678-687.

Goldschmidt M, L Peña, R Rasotto, V Zappulli. 2011. Classification and grading of canine mammary tumors. Vet Pathol 48, 117-131.

Im KS, NH Kim, HY Lim, HW Kim, JI Shin, JH Sur. 2014. Analysis of a New Histological and Molecular-Based Classification of Canine Mammary Neoplasia. Vet Pathol 51, 549-559.

Millanta F, M Calandrella, G Bari, M Niccolini, I Vannozzi, A Poli. 2005. Comparison of steroid receptor expression in normal, dysplastic, and neoplastic canine and feline mammary tissues. Res Vet Sci 79, 225-232.

Moe L. 2001. Population-based incidence of mammary tumours in some dog breeds. J Reprod Fertil Suppl 57, 439-443.

Mulligan RM. 1975. Mammary cancer in the dog: a study of 120 cases. Am J Vet Res 36, 1391-1396.
Priester WA, FW McKay. 1980. The occurrence of tumors in domestic animals. Natl Cancer Inst Monogr 54, 1-210.

Richards HG, PE McNeil, H Thompson, SWJ Reid. 2001. An epidemiological analysis of a canine-biopsies database compiled by a diagnostic histopathology service. Prev Vet Med 51, 125-136.

Saba CF, KS Rogers, SJ Newman, GE Mauldin, DM Vail. 2007. Mammary gland tumors in male dogs. J Vet Intern Med 21, 1056-1059.

Sleeckx N, H de Rooster, EJ Veldhuis Kroeze, C Van Ginneken, L Van Brantegem. 2011. Canine mammary tumours, an overview. Reprod Domest Anim 46, 1112-1131.

Sorenmo K. 2003. Canine mammary gland tumors. Vet Clin North Am Small Anim Pract 33, 573-596.

Torres CG, AM Pino, WD Sierralta. 2009. A cyclized peptide derived from alpha fetoprotein inhibits the proliferation of ER-positive canine mammary cancer cells. Oncology Rep 21, 1397-1404.

Vinothini G, RS Murugan, S Nagini. 2009. Evaluation of molecular markers in a rat model of mammary carcinogenesis. Oncol Res 17,483-493.

Zatloukal J, J Lorenzova, F Tichy, A Necas, H Kecova, P Kohout. 2005. Breed and age as risk factors for canine mammary tumours. Acta Veterinaria 74, 103-109. 
